\title{
Unruhige Kinder und pflanzliche Sedativa - Erfahrungen aus der Praxis
}

\author{
Walter Dorsch \\ München, Deutschland
}

In fast allen Situationen, in denen wegen des Symptoms «unruhiges Kind» der Einsatz von Medikamenten sinnvoll oder notwendig erscheint, sind pflanzliche Arzneimittel synthetischen vorzuziehen, und zwar wegen ihres durchweg günstigeren Nebenwirkungsprofils und ihrer schwächeren und deshalb besser steuerbaren Wirkung.

Eine ausschliesslich pharmakologische Behandlung ist immer zum Scheitern verurteilt, auch wenn sich genervte Eltern noch so sehr einen schlagartig wirkenden Beruhigungstee für den brüllenden Säugling oder eine Pille wünschen, die ihren aufsässig pubertierenden, «hyperkinetischen» Sprössling ruhig stellt und ihm nebenher gute Schulnoten verschafft.

Normale Kinder sind immer mehr oder weniger unruhig. Die Toleranz gegenüber (unruhigen) Kindern ist sehr variabel, auch die Definition «Unruhe».

Im Säuglingsalter ist die Symbiose zwischen Mutter und Kind oft so eng, dass auch aus nichtigem Anlass ein Teufelskreis entstehen kann, der nur schwer zu durchbrechen ist. Aus der Perspektive des Säuglings dürften Nervosität und Unsicherheit der Eltern die häufigsten Ursachen von Unruhe sein, gefolgt von dem Gefühl, nicht richtig oder nicht rechtzeitig verstanden zu werden. Daneben gibt es natürlich eine grosse Zahl körperlicher Beschwerden, die lang anhaltende Unruhezustände zur Folge haben - wie chronischer Hunger durch Mangelernährung oder Nahrungsmittelverwertungsstörungen, Stillprobleme, Verdauungsstörungen, Blähungen, Koliken, intraabdominelle Fehlbildungen, Leistenbruchanlagen mit rezidivierenden Einklemmungen und andere mehr. Wenn elementare Bedürfnisse nicht befriedigt werden, kann die Wut des Säuglings tödlich werden und vor allem seelisch instabile Eltern zu unbedachten Handlungen bringen. Seitens der Mutter stehen oft Versagensängste und eine allgemeine Hilflosigkeit im Vordergrund, die bis zur Umkehr der Rollenverteilung zwischen Mutter und Kind führen kann und oft durch divergente Ratschläge und Besserwisserei der nächsten sozialen Umgebung verstärkt wird. Wochenbettpsychosen dürfen nicht übersehen werden.
Bei älteren Kleinkindern treten andere körperliche Ursachen wie Laktoseintoleranz, Nahrungsmittelallergien und Glutenenteropathie in den Vordergrund. Auch sind unruhestiftende Erziehungsfehler zu beobachten wie Überforderung durch Überförderung, Ruhigstellen durch stundenlanges Fernsehen, ungebremster Gebrauch elektronischer Medien, Vernachlässigung, auch Wohlstandsvernachlässigung, und grenzenloses Verwöhnen. Manche Eltern vermitteln ihren Kindern das Gefühl, sie in ihren Fragen und Konflikten nicht ernst zu nehmen. Manche Kinder wiederum werden täglich mit Hunderten von Anordnungen konfrontiert, die sie - wen wundert es? - weder verarbeiten noch befolgen können.

Schulkinder sind oft noch stärkeren unruhestiftenden Stressfaktoren ausgesetzt: übergrosse Leistungsanforderungen, unzureichende Unterweisung und Führung, Ehrgeiz der Eltern, Mobbing und viele andere.

Die Welt, in die wir unsere Jugendlichen entlassen, ist unruhig. Wer wundert sich über unruhige Jugendliche, vor allem wenn der pubertätsspezifische Hormonsturm über sie hereinbricht und sie sich überall zurechtfinden müssen - in der Schule, in oft schwierigen Familien, in den diversen sozialen Netzwerken mit deren Konformitätsdruck, entgegen der bewusst Sucht erzeugenden Produkte der elektronischen Unterhaltungsindustrie? Manche latente Depression wird verkannt, (noch) normales Verhalten als Aufmerksamkeitsdefizit-/Hyperaktivitätsstörung (ADHS) denunziert.

Der Stellenwert pflanzlicher Arzneimittel bei einer Auswahl alltäglicher pädiatrischer Probleme soll dargestellt werden. Die Praxis hat ergeben, dass oft auch vermeintlich Banales erklärt und gezeigt werden muss. Beim Säugling bleibt auch eine ausführliche Anamnese und sorgfältige Untersuchung oft ohne spezifisches Ergebnis, sodass ein Arzt oder auch ein nichtärztlicher Betreuer zunächst unspezifische Massnahmen ergreifen wird:

- Vor allen Handgriffen am Säugling: innere Ruhe herstellen;

- langsames, behutsames Handeln (z.B. Wiegen statt Schütteln);

\section{KARGER}

Fax +497614520714

Information@Karger.com

www.karger.com
Prof. Dr. med. Walter Dorsch

Facharzt für Kinderheilkunde und Jugendmedizin, Naturheilverfahren, Allergologie und Kinderpneumologie

Aidenbachstrasse 118, 81379 München, Deutschland

walter_dorsch@web.de 
Abb. 1. Die Hockhaltung ist oft hilfreich, insbesondere bei Bauchschmerzen und Blähungen.

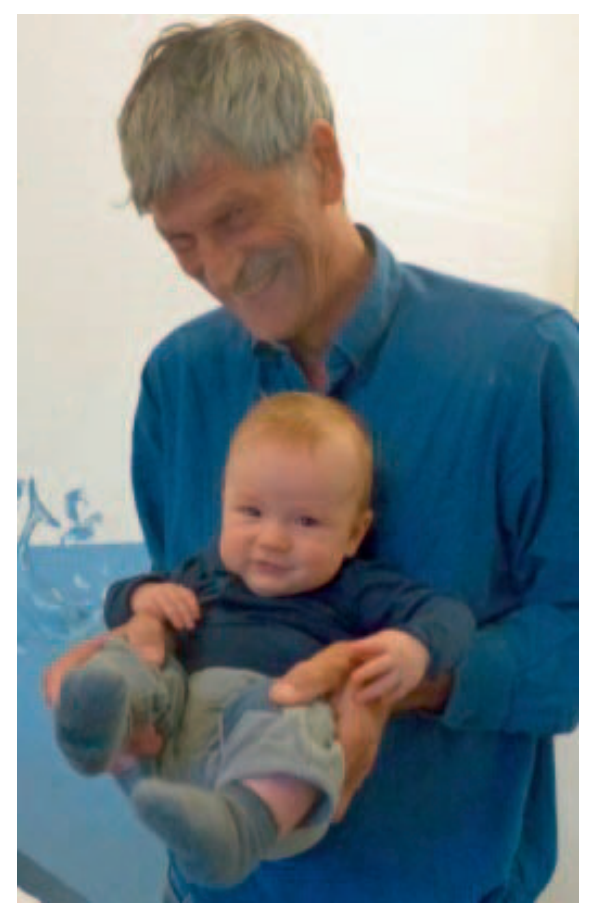

- keine Ablenkungs- oder Beruhigungsmanöver im Sekundentakt;

- (möglichst) strukturierter Tagesablauf;

- feste Rituale beim Stillen;

- aktives Zuhören;

- intensive Zuwendung während der anfangs kurzen Aktivitätsphasen;

- feste Einschlafrituale.

Beim Einschlafen empfiehlt sich ein möglichst kurzes, regelhaft und gleichmässig ablaufendes Ritual, das durch pflanzliche Arzneimittel ergänzt werden kann und sollte: Melissenbad, Baldriantropfen, eventuell ein Hopfenkissen oder diverse Kombinationspräparate.

Bei Blähungen und Bauchschmerzen stellt die Pflanzenheilkunde Kümmelöl und diverse Windsalben, die im Uhrzeigersinn einmassiert werden, zur Verfügung. Vor allem hastig trinkende Säuglinge schlucken sehr viel Luft. Zum Aufstossen gibt es viele empfohlene Handgriffe (Abb. 1); es wird erleichtert durch Polisiloxan, das unmittelbar vor dem Trinken (und nicht im Fläschchen) verabreicht werden sollte.

Störungen der Mutter-Kind-Interaktion sind nicht selten. Betroffen sind oft junge Mütter, die sich alleingelassen und überfordert fühlen. Schuldzuweisungen oder auch versteckter Tadel sind möglichst zu vermeiden. Oft reicht es, wenn
Tab. 1. Beispiele von bewährten Teemischungen nach Dorsch

\begin{tabular}{ll}
\hline Rezept 1 & \\
Baldrianwurzel & $40,0 \mathrm{~g}$ \\
Passionsblumenkraut & $30,0 \mathrm{~g}$ \\
Melissenblätter & $30,0 \mathrm{~g}$ \\
\hline Rezept 2 & \\
Baldrianwurzel & $20,0 \mathrm{~g}$ \\
Hopfenzapfen & $10,0 \mathrm{~g}$ \\
Fenchelsamen (ang.) & $10,0 \mathrm{~g}$ \\
Lavendelblüten & $10,0 \mathrm{~g}$ \\
\hline
\end{tabular}

es gelingt, Gelassenheit und Freude am Kind zu vermitteln. Zur allgemeinen Beruhigung können pflanzliche Sedativa adjuvant eingesetzt werden. Für den Säugling geeignet sind: Melissenblätter, Lavendelblüten, auch Baldrian. Baldrian hat keine spezifische Verwendung bei stillenden Müttern, wird aber am häufigsten verwendet, um Angst und Schlafstörungen zu behandeln. Gelegentlich wird er auch zur eigenverantwortlichen Behandlung von postpartalen Stimmungstiefs oder Depressionen verwendet.

Normale Kleinkinder sind voller Tatendrang und lassen sich auch durch (pflanzliche) Arzneimittel, deren Anwendung bei dieser Indikation verantwortet werden kann, oft kaum bändigen, aber Baldrian, Hopfen und Melisse können als Einschlafhilfe bzw. Sedativa auch in der Form von Tees (Tab. 1) eingesetzt werden.

Dem Schulkind hilft kein Medikament beim Stillsitzen. Disziplin muss gelernt und geübt werden. Hilfreich ist eine Strukturierung des Tagesablaufs im Sinne der Kneipp'schen Ordnungstherapie. Schulleistungen bessern sich unter konsequenter Anwendung der Progressiven Muskelrelaxation nach Jacobson. Baldrian kann als Einschlafhilfe sinnvoll sein, und Hopfen hilft ein wenig, ohne die Aufmerksamkeit stark einzuschränken. Pflanzliche Arzneimittel sind bei eindeutigem $\mathrm{AD}(\mathrm{H}) \mathrm{S}$ sicher nicht ausreichend wirksam.

\section{Fazit}

Bei der Behandlung unruhiger Kinder, genauer: von Familien mit unruhigen Kindern, spielen pflanzliche Arzneimittel eine überaus wichtige, aber selten die erste Rolle. In fast allen Belangen sind sie synthetischen Arzneimitteln überlegen.

\section{Disclosure Statement}

Es besteht kein Interessenkonflikt. 